\title{
Neuroprotective Activity of Protocatechuic Acid (3,4-Dihydroxybenzoic Acid) to Treat Parkinson's Disease
}

\author{
Jagatheesh Kaliaperumal ${ }^{1}$, Satguru Dulari², A. Pandurangan ${ }^{3}$, Mahendra Singh Rathore ${ }^{4}$, \\ Amit Mittal ${ }^{5}$, Md. Shamshir Alam ${ }^{6}$
}

\begin{abstract}
${ }^{1}$ Department of Pharmacy, Maharishi Markandeshwar (Deemed to Be University), Mullana, Haryana, India. ${ }^{2}$ Department of Pharmacy, Maharishi Markandeshwar (Deemed to Be University), Mullana, Haryana, India. ${ }^{3}$ Department of Pharmacy, Maharishi Markandeshwar (Deemed to Be University), Mullana, Haryana, India. ${ }^{4}$ Department of Pharmacy, Maharishi Markandeshwar (Deemed to Be University), Mullana, Haryana, India. ${ }^{5}$ MMIMSR, Maharishi Markandeshwar (Deemed to Be University), Mullana, Haryana, India. ${ }^{6}$ Department of Pharmacy, Maharishi Markandeshwar (Deemed to Be University), Mullana, Haryana, India.
\end{abstract}

\section{ABSTRACT}

\section{BACKGROUND}

The present study was designed to explore the possible neuroprotective role of protocatechuic acid (3,4-dihydroxybenzoic acid) against rotenone induced locomotor, oxidative and mitochondrial dysfunction in mice. We also wanted to evaluate its ability to prevent neuronal degeneration in rotenone intoxicated mice.

\section{METHODS}

Thirty mice, Albino strain, between $20-25$ g, were included in the study. Group 1 is control group. It received sunflower oil $0.1 \mathrm{~mL}$ subcutaneously for two weeks. Group 2 received rotenone $(1 \mathrm{mg} / \mathrm{Kg}$ ) S.C. Group 3 also received rotenone (1 mg / Kg / day) S.C. in sunflower oil alternatively and Protocatechuic acid $10 \mathrm{mg} / \mathrm{Kg}$ B.W. P.O. for 2 weeks. Group 4 and 5 received rotenone as same in group 2 and Protocatechuic acid (25 mg / Kg) and (50 mg / Kg) B.W. P.O. After the finish of trial, open field test, stride length measurement, catalepsy, forced swim test, sucrose preference test and elevated plus maze test were conducted.

\section{RESULTS}

Exposure to Rotenone in mice led to reduction in peripheral and central movements. Pre-treatment with protocatechuic acid prior to rotenone exposure was able to maintain the both motor and non-motor symptoms of PD. However, protocatechuic acid was effective in the maintenance of dopaminergic and nondopaminergic pathways.

\section{CONCLUSIONS}

This study strengthens the fact that the protocatechuic acid in rotenone-affected areas might offer neuroprotection.
Corresponding Author: Dr. Jagatheesh Kaliaperumal, Professor,

MM College of Pharmacy, Maharishi Markandeshwar, (Deemed to Be University), Mullana, Haryana, India. E-mail: kjagatheesh@gmail.com

DOI: $10.14260 / \mathrm{jemds} / 2020 / 536$

How to Cite This Article: Kaliaperumal J, Dulari S, Pandurangan A, et al. Neuroprotective activity of protocatechuic acid (3,4-dihydroxybenzoic acid) to treat Parkinson's disease. J Evolution Med Dent Sci 2020;9(34):24662471, DOI: $10.14260 / \mathrm{jemds} / 2020 / 536$

Submission 31-10-2019,

Peer Review 14-07-2020,

Acceptance 20-07-2020,

Published 24-08-2020.

Copyright (C) 2020 JEMDS. This is an open access article distributed under Creative Commons Attribution License [Attribution 4.0 International (CC BY 4.0)]

\section{KEY WORDS}

Explore, Counteractive, Peripheral 


\section{BACKGROUND}

Parkinson's syndrome is a dynamic neurological malady.[1] Parkinson's ailment manifestations may incorporate resting tremor, balance issues, and motor capacities, just as issues in cerebral capacity.[2] Parkinson's ailment influences 100-300 individuals from 100.000 people, and is the most well-known neurodegenerative illness after Alzheimer's. The expense in Europe is 798 billion euros every year. [3] PD makes dopamine generation decline in a portion of the mind called considerable nigra, which is a portion of basal ganglia in the cerebrum. ${ }^{[4]}$ The basal ganglia are one of the working units of the cerebrum in connection to locomotive control. At the point when the basal ganglia don't function may be it should arise PD side effects. The side effects normally incorporate troubles in development and capacity among different issues.[5] As nerve cells in parts of the mind become hindered or expire, individuals may start to see issues with development, tremor, difficulty in the extremities or the storage compartment of the body, or decreased balance. As these side effects become increases, individuals may experience issues take a turn, talking, or finishing other basic assignments. Most cases are sporadic that is, the infection does not ordinarily keep running in families. It is felt that PD likely outcomes from a blend of hereditary vulnerability and presentation to at least one obscure natural factors that trigger the illness. While most types of Parkinsonism have no known reason, there are cases in which the reason is known or suspected or where the side effects result from another syndrome. No solution for PD exists today, however research is continuous and meds or medical procedure can regularly give generous improvement.[6] Parkinson's ailment (PD) is an unending dynamic neurodegenerative issue described by expiry of dopaminergic neurons in the significant nigra standards compacta (SNpc) and wide spread of alpha synuclein, an intracellular protein. Dopamine lack in the basal ganglia prompts bradykinesia, tremor, inflexibility and later postural shakiness. These nonmotor manifestations become serious indications in the later phases of PD. Many impairments grow later throughout the illness including non-motor side effects, dopamine and motor problems of long dopamine treatment.

Despite the fact that there have been advances in the curative and clinical treatment for PD, but complete disease altering treatment is not available.[7] As indicated by Parkinson's infection Foundation, Parkinson's malady is a perpetual development issue brought about by breaking down motor spots where side effects proceed and decline after some time. The focal sensory system of an individual is influenced by the malady where the neurons can't transmit data among themselves, bringing about inflexible and moderate or even nonattendance of physical development.[8] The principal portrayal of Parkinson's illness (PD) was given by James Parkinson in mid nineteenth century. ${ }^{[9]}$ However, the information about this sickness has been available in India since earliest. Despite the fact that the frequency of PD in India is less related with different nations, ${ }^{[10]}$ the all-out weight of PD is a lot higher because of huge public. We assessed all the distributed writing on PD from India till date. This will help in giving a complete viewpoint with respect to the past and the momentum circumstance of this sickness in our nation.[11] Past research recommends that $\mathrm{PD}$ as a rule, influences individuals after the age of 50 years. Just $10 \%$ of patients with PD report indications before the age of 40 years. In addition, PD is evaluated to influence $1.6 \%$ of people beyond 65 years old years. Given away that age is the most significant factor for beginning of $\mathrm{PD}$, and that the people is becoming older, the insights for the quantity of influenced people get proliferate sooner. It is evaluated that up to $90 \%$ of patients with PD create hypokinetic dysarthria over the period of their ailment. This change of speech may likewise be probably the soonest pointer of the sickness. For grown-up peoples, strategies permitting the evaluation of the speech execution and acoustic criticism tests can be basic for increasing inspiration and ability for language training. At the end of the day, acoustic estimations can improve the individual treatment, and hence incompletely lighten the bother and cost of physical visits.

In addition, voice estimation is non-obtrusive, inexpensive and easy to oversee. PD-related side effects advance in a period subordinate way to such an extent, that the presymptomatic stages are related with smell and autonomic dysfunctions, the symptomatic stages with motor variations from the norm, and the last stages with dementia. The moderate movement of the infection results from neurodegeneration burdening the olfactory bulb and the medulla oblongata in pre-symptomatic stages 1 and 2, the SNpc in stages 3 and 4, and the epencephalic cortex in stages 5 and 6.[12] The way that PD patients likewise experience nonmotor cerebral, and autonomic side effects demonstrates that non-dopaminergic neurons additionally turned out to be influenced over the span of the ailment.[13] The illness happens more habitually in men than in ladies in each time of life, which is clarified by the neuroprotective impacts of oestrogens. Different types of Measurement tools also used to calculate, aimed the behaviour of PD patients. These tools useful for determination of problems for example mobility of patient, their body language, their involvement and tasks perform. These tools helpful to relief the patient by encourage patient, recover them, and identify illness. These tools are used according to the condition of patient. Other diseases like depression, anxiety also occur in PD patients.[14] Protocatechuic acid (3,4-dihydroxybenzoic acid) have surely antioxidant and metal chelating properties[15] and it can be fundamentally changed that can give extra biological action. It is a basic phenolic acid. It is found in a huge range of consumable plants and has different pharmacological actions. This bioactive compound is well known for its organic properties and pharmacological actions, for example, cancer prevention agent, antibacterial, anticancer, antiulcer, ant diabetic, antiaging, ant fibrotic, antiviral, mitigating, pain relieving, ant atherosclerotic, heart, hepatoprotective, neurological and nephroprotective.

The neuroprotective impacts of PCA, removed from Alpinia oxyphylla.[16] Apoptotic cell demise by $\mathrm{H}_{2} \mathrm{O}_{2}$ was dose dependent. Improved impact of PCA on securing PC12 cells against apoptosis, expanded glutathione (GSH) level and an expansion in reactant movement was examined by stream cytometric examination. In this way, PCA can be valuable for treating asthma. Exploratory examinations emphatically bolster the job of protocatechuic corrosive in the counteractive action of neurodegenerative procedures, including Alzheimer's and Parkinson's infections, because of its great effect on procedures basic intellectual and conduct hindrance, to be specific collection of the $\beta$-amyloid plaques in mind tissues, hyper phosphorylation of tau protein in neurons, 
unreasonable development of receptive oxygen species and neuroinflammation.[17]

\section{METHODS}

\section{Chemicals}

Rotenone obtained from Sigma-Aldrich, (Bengaluru, India) and used to induced Parkinson's or neurodegeneration respectively. The sunflower oil is also used to dissolve rotenone and as a vehicle.

\section{Animals and Drug Treatment}

Albino Mice were randomly divided into 5 equal groups, with 6 mice in each group. Group 1 is control received sunflower oil $0.1 \mathrm{~mL}$ subcutaneously for two weeks. In group 2 alternatively administered rotenone (1 mg / Kg) subcutaneously. In Group 3 mice treated with rotenone (1 $\mathrm{mg} / \mathrm{Kg} /$ day) subcutaneously in sunflower oil alternatively and also administer 3,4Dihydroxybenzoic acid $10 \mathrm{mg} / \mathrm{Kg}$ B.W. P.O. for 2 weeks. In group 4 administered rotenone as same in group 2 and 3,4Dihydroxybenzoic acid ( $25 \mathrm{mg} / \mathrm{Kg}$ ) B.W. P.O. In group 5 also administered rotenone as same in group 2 and 3,4Dihydroxybenzoic acid (50 mg / Kg) B.W. P.O. After the finish of the trial time conduct tests, for example, open field test, stride length measurement, catalepsy, forced swim test, sucrose preference test and elevated plus maze test were performed.

\section{Motor Behavioural Tests}

Open Field Test - The floor of the apparatus cover with white paper and lines were drawn i.e. 20 equal squares $(15 \mathrm{~cm} \times$ $15 \mathrm{~cm})$. Animals were placed at the centre and their behaviour were detected. The number of lines crossing measured the loco motor activity i.e. four paws needed to cross the line to be counted as line crossing and as with the latency to leave central square or time spend in Central Square, also licking scratching were measured. The apparatus cleaned with alcohol and dried in each session.

Stride Length Measurement - Take a clean white sheet, then trained the animals to walk along straight line. Next day, the forepaws of mice were dipped in the blue ink, then allowed them to walk on the clean sheet. Then measure their foot prints distance of the same side of animal. Then measure stride length from the middle toe of $1^{\text {st }}$ step to the heel of 2 nd step.

Catalepsy - This test detected the immobility behaviour of animals i.e. the rigidity of muscles. In this the animals lifted with tail and their forepaws placed on the horizontal bar having $10 \mathrm{~cm}$ height and stopwatch was started for $180 \mathrm{~s}$. Then detect the first movement of paws which is called as cataleptic time.

\section{Non-Motor Behavioural Tests}

Modified Forced Swim Test - Firstly trained the animals for swim, put the animals in water tank for $15 \mathrm{~min}$. Then next day animals were allowed to swim for 5 minutes. In this test mice were dropped into inescapable beaker filled with water. They were forced to swim until they were closed to drowning. The animals paddled furiously until they eventually give up and floated. The amount of time that the animal spend struggling vs floating was then measured. The depressed animals give up earlier. Water of tank must be changed after each animal to avoid the smell.

Sucrose Preference Test - Firstly mice were transferred into single cage without any food. Also trained the mice to drink water from two bottles on both sides. Then next day, one water bottle placed and $2^{\text {nd }}$ bottle which contain $1 \%$ sucrose was placed for $1 \mathrm{hr}$ and after $24 \mathrm{~h}$ later the bottles were reversed. Then calculate the water and sucrose consumption known as total consumption. The percentage of sucrose intake was calculated as (\% sucrose preference $=$ sucrose intake $\times 100 /$ total intake). After this test, all mice received free access to food and water.

Elevated Plus Maze Test - The elevated plus maze consists of four arms arranged around a small central platform in a plus shape. Two arms were enclosed with high walls and the other two arms were open. The animals were placed in the central platform facing one of the open arms. The animal was allowed to explore the maze for a specific amount of time. A timer is used to calculate how much time it spends on each of the arms. The number of entries into the open and closed arms were recorded as well as the time spent in each arm. Typically, animals had greater number of arm entries into closed arms than open arm due to their natural avoidance of open spaces.

\section{RESULTS}

\section{Effects of Protocatechuic Acid on Rotenone Induced} Locomotor Activity

The loco motor and non-loco motor activities of the animals were observed by open field test. Group $4^{\text {th }}$ (Rotenone subcutaneously) showed reduction in loco motor and non-loco motor activity. Whereas group $1^{\text {st }}$ and $2^{\text {nd }}(3,4-$ dihydroxybenzoic acid orally) showed increase in loco motor and non-loco motor activity as compared to group 4, as shown in figure 1. Also, no significance changes were found in control group 3 (sunflower oil subcutaneously) and group ( $1^{\text {st }}$ and $\left.2^{\text {nd }}\right)$.

\section{Effects of Protocatechuic Acid on Rotenone Induced Stride Length Measurement}

The distance between forepaws of mice were observed by stride length measurement. The result of this test showed the group 4 (rotenone subcutaneously) showed decrease in forelimb stride length. Whereas, group $1^{\text {st }}$ and $2^{\text {nd }}(3,4-$ dihydroxybenzoic acid orally) showed increase in forelimb stride length, as shown in figure 2. In Addition, no significance changes were found in stride length of control group 3 (sunflower oil subcutaneously) and group ( $1^{\text {st }}$ and $\left.2^{\text {nd }}\right)$. 
Effects of Protocatechuic Acid on Rotenone Induced Movement Impairments

The muscle rigidity and impairing movement was detected by this test. The result of this test observed that the group 4 (rotenone subcutaneously) showed impaired posture as compare to control group. Whereas group $1^{\text {st }}$ and $2^{\text {nd }}(3,4-$ dihydroxybenzoic acid orally) showed less catalepsy as compared to group 4, as shown in figure 3. In Addition, no significance changes were found in stride length of control group 3 (sunflower oil subcutaneously) and group (1 $1^{\text {st }}$ and $2^{\text {nd }}$.
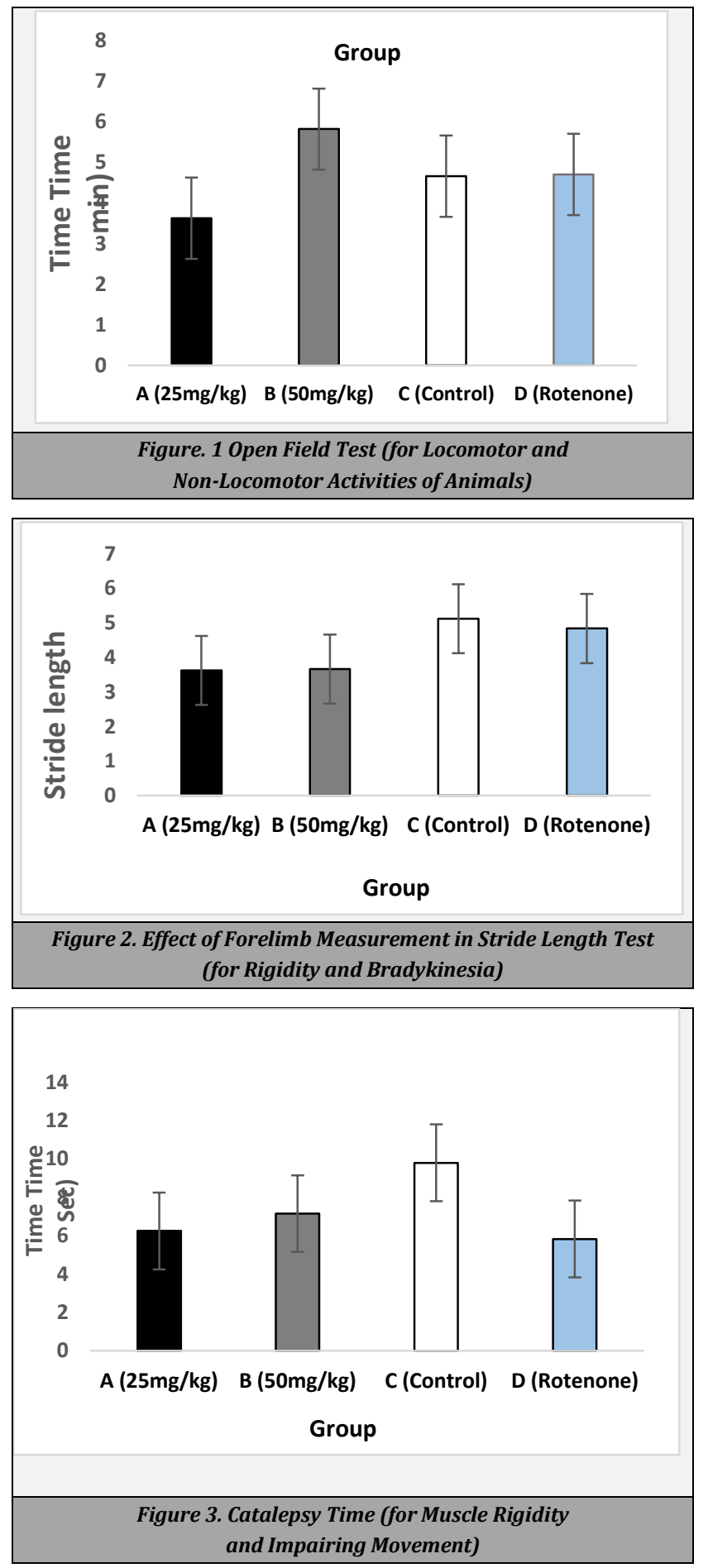
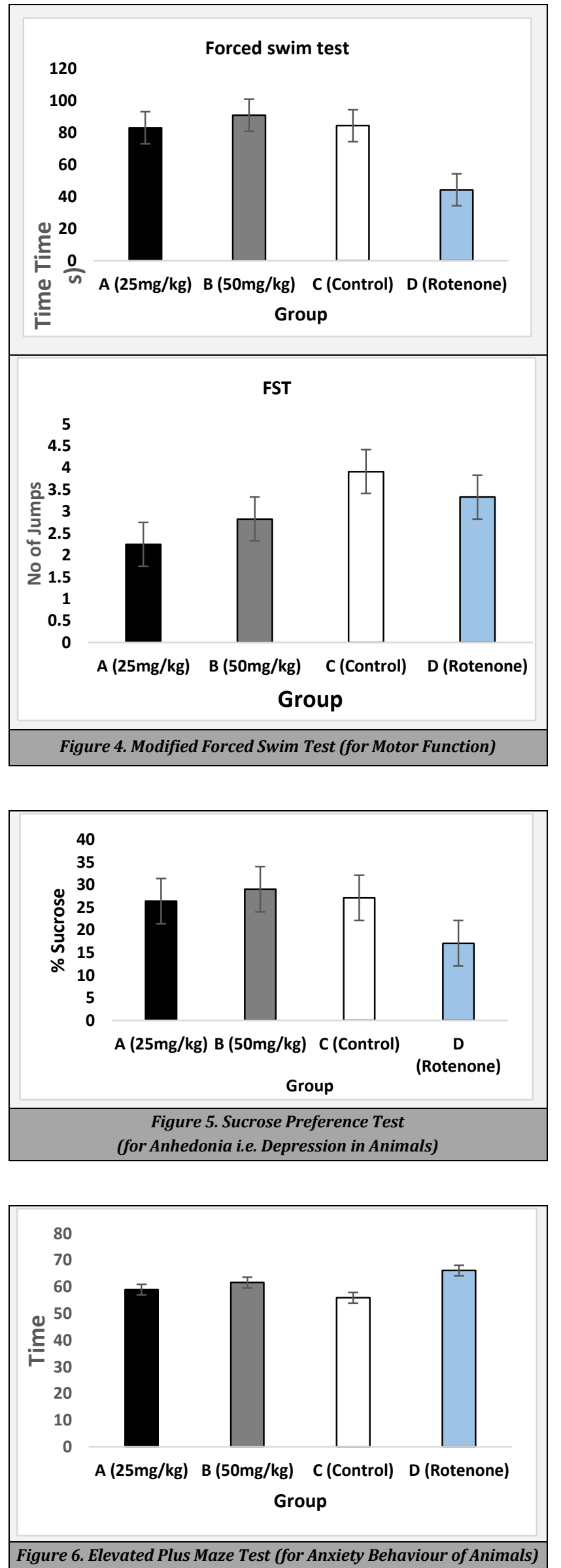


\section{Effects of Protocatechuic Acid on Rotenone Induced Forced Swim Test}

The immobility showed the depressive behaviour of mice. The result of this test observed that the group 4 (rotenone subcutaneously) showed more immobility in water tank as compared to control mice. In addition, group $1^{\text {st }}$ and $2^{\text {nd }}(3,4-$ dihydroxybenzoic acid orally) showed less immobility time as compared to group 4, as shown in figure 4. In Addition, no significance changes were found in stride length of control group 3 (sunflower oil subcutaneously) and group (1 $1^{\text {st }}$ and $2^{\text {nd }}$.

\section{Effects of Protocatechuic Acid on Rotenone Induced Sucrose Preference Test}

The result of this test observed that the group 4 (rotenone subcutaneously) showed significant decrease in the intake of sucrose water as compared to compared group, as shown in figure 5 . In addition, group 1st and 2nd (3,4-dihydroxybenzoic acid orally) showed improved in sucrose intake. The sugar intake test indicates depressive like behaviour. In Addition, no significance changes were found in stride length of control group 3 (sunflower oil subcutaneously) and group (1st and 2nd).

\section{Effects of Protocatechuic Acid on Rotenone Induced Elevated Plus Maze Test}

The anxiety behaviour observed in this test. The result of this test observed that the group 4 (rotenone subcutaneously) spend more time in enclosed arm than the open arm as compared to group 3, as shown in figure 6. In addition, group $1^{\text {st }}$ and $2^{\text {nd }}$ (3,4-dihydroxybenzoic acid orally) showed improved the anxiety like behaviour. In Addition, no significance changes were found in stride length of control group 3 (sunflower oil subcutaneously) and group (1 $1^{\text {st }}$ and $2^{\text {nd)}) .}$

\section{DISCUSSION}

The open field test, stride length measurement and catalepsy test are utilized to survey the loco motor activity, though sucrose consumption, elevated plus maze test and forced swim test are utilized to survey the non-motor hindrances including anhedonia, and uneasiness in mice. When rotenone administered to the mice, this led to reduction in motor and non-motor works when contrasted with control mice. In open field test, the number of lines crossed shows the peripheral loco motor activity, the number of the time rat standing alone with its fore legs shows rearing activity and the number of times the rat washing face or scratching or licking the fur shows grooming activity. Decreased performance of these exercises in rotenone stimulated creatures could be related with dopaminergic depletion. Stride length estimation used to test the bradykinesia and rigidity, a significant decrease in forelimb stride length was seen in rotenone induced mice. The beings with PD present a reduced walk length with a mix up step and moderated speed (progress hypokinesia). In catalepsy, the duration taken for the first movement of paws was measured as cataleptic time and the administration of rotenone led to the inability of posture. Rotenone destroyed the dopaminergic neurons specifically and bringing about inhibited engine work. In this study, reduction of cerebrum DA levels in rotenone treated mice caused behaviour variations from the mean as found in patients with PD. Improvement of striatal DA by Protocatechuic acid clearly showed the neuroprotective proficiency, likewise securing dopaminergic neurons and subsequently normalizing the behaviour. In swim test, Rotenone-administered mice showed more immobility time in forced swim test as compared to control mice, because rotenone destroy the dopaminergic neurons so produce motor dysfunction. The reduction of DA levels in rotenone-treated mice caused social defects as found in patients with PD. The improvement of striatal DA due to neuroprotective behaviour of Protocatechuic acid shielding the dopaminergic neurons. Sucrose inclination useful to check anhedonia i.e. depression in mice. Depletion of neurotransmitters by rotenone prompts depressive behaviour, while protocatechuic acid offers neuroprotective impact that might be because of its stimulant activity. Destructions of dopaminergic, serotonergic and noradrenergic contexts have been assumed the sadness in PD. In elevated plus maze test, Anxiety-like behaviour was measure, mice treated with rotenone spend more time in the enclosed arms than the open arms as compared to control mice. Rotenone treatment altered both the motor and nonmotor behavioural function as compared to controls. The cholinergic context assumes a vital job in learning and memory related capacities. Additionally increment in AChE program may be responsible for diminished memory in rotenone treated mice. Concurrent treatment with protocatechuic acid significantly restored these behavioural deficits.

\section{CONCLUSIONS}

Exposure to Rotenone in mice led to reduction in peripheral and central movements. Rotenone neurotoxicity resulted in loss of dopaminergic neurons in SN, mitochondrial dysfunction, depression, fatigue etc. Pre-treatment with protocatechuic acid prior to rotenone exposure was able to maintain both motor and non-motor symptoms of PD in the cerebral cortex, olfactory bulb and cerebellum. However, protocatechuic acid was effective in the maintenance of dopaminergic and nondopaminergic pathways. Therefore, protocatechuic acid treatment has a potential to manage the motor and cognitive function in early PD and delay the deterioration of motor and cognitive function in PD patients. Results of this study strengthen the fact that the protocatechuic acid in rotenone-affected areas might offer neuroprotection.

Financial or Other Competing Interests: None.

\section{REFERENCES}

[1] Findley LJ. The economic impact of Parkinson's disease. Parkinsonism Relat Disord 2007;13 Suppl:S8-12. 
[2] Morris M, Iansek R. Characteristics of motor disturbance in Parkinson's disease and strategies for movement rehabilitation. Human Movement Science 1996;15(5):649-69.

[3] Olesen J, Gustavsson A, Svensson M, et al. The economic cost of brain disorders in Europe. Eur Jo Neurol 2010;19(1):155-62.

[4] Pendey S, Singh B, Verma R, et al. Current Understanding of Parkinson's disease. International Journal of Pharmacotherapy 2013;3(2):70-9.

[5] Nykänen M. Psychometric evaluation of Parkinson's disease measurement tools for Toimia network: modified Parkinson activity scale and push and release test. Degree Programme in Physiotherapy 2016.

[6] Office of Communications and Public Liaison. National institute of neurological disorders and stroke. National Institutes of Health, Bethesda. MD 20892.

[7] Radhakrishnan DM, Vinay G. Parkinson's disease: a review. Neurology India, Neurological Society of India 2018;66(7):26-35.

[8] Burbulla LF, Song P, Mazzulli JR, et al. Dopamine oxidation mediates mitochondrial and lysosomal dysfunction in Parkinson's disease. Science 2017;357(6357):1255-61.

[9] Pearce JM. Aspects of the history of Parkinson's disease. J Neurol Neurosurg Psychiatry 1989;Suppl:6-10.

[10] Singhal B, Lalkaka J, Sankhla C. Epidemiology and treatment of Parkinson's disease in India. Parkinsonism Relate Disorder 2003;9(Suppl 2)105-9.
[11] Surathi P, Jhunjhunwala K, Yadav R. Research in Parkinson's disease in India: a review. Ann Indian Acad Neurol 2016;19(1):9-20.

[12] Braak H, Tredici KD, Bratzke $H$, et al. Staging of the intracerebral inclusion body pathology associated with idiopathic Parkinson's disease (preclinical and clinical stages). J Neurol 2002;249 Suppl 3:1-5.

[13] Al-Maqtari T. Approaches in the treatment of Parkinson's disease: a focus on stem cell- based therapies. Electronic Theses and Dissertations Paper 34, 2012.

[14] Dubayova T. Parkinson's disease - psychological determinants of quality of life. University of Groningen 2010.

[15] Ramassamy C. Emerging role of polyphenolic compounds in the treatment of neurodegenerative diseases: a review of their intracellular targets. Eur J Pharmacol 2006;545(1):51-64.

[16] Ordoudi SA, Tsimidou MZ, Vafiadis AP, et al. StructureDPPH scavenging activity relationships: parallel study of catechol and guaiacol acid derivatives. J Agric Food Chem 2006;54(16):5763-8.

[17] Mandel S, Amit T, Bar-Am 0, et al. Iron dysregulation in Alzheimer's disease: multimodal brain permeable iron chelating drugs, possessing neuroprotective-neurorescue and amyloid precursor protein-processing regulatory activities as therapeutic agents. Prog Neurobiol 2007;82(6):348-60. 